death, which occurred on the fourth day, tense and tympanitic.

A milkman, aged twenty-eight, who had been subject to rupture ever since he was five years old, after having had a heavy day in carrying milk about, and immediately after a violent fit of laughing, found that his rupture had come down, and that he could not push it back as he had been in the habit of doing. He went to a surgeon, who reduced it, "after having worked at it for half an hour." He had pain in the stomach before and after reduction. The symptoms of strangulation were rather aggravated than relieved, and continued so for forty-four hours, when he was admitted. There was no evident tumour in the inguinal canal, the cord, on the contrary, being very distinctly felt, and having no sac either in front or behind it, below the level of the outer ring. As some obscurity existed about the case, the operation was deferred for twenty hours to give trial to palliative treatment, which, however, was of no advantage. The inguinal canal was laid open in the direction of its long axis, and when fully exposed, there was remarked at the upper part, the cord thrown forwards, and spread over a tumour below and behind it, on a level with the inner ring. The cord was covered by a rather dense fascia, and after having been drawn aside, the sac was opened. It contained about six inches of dark intestine. The neck of the sac was behind the level of the inner ring, which was nearer than usual to the outer. Although in this case the taxis had brought about the nearly complete reduction of the hernia en bloc, the temporary disappearance of the gut from the grasp of one surgeon prevented any further manipulation and mischief, and probably saved the life of the patient, who, fortunately, recovered on the fifteenth day, under the treatment of another.

(To be continued.)

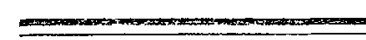

ON

SOME OF THE RARER AND MORE IMPORTANT

\section{CASES IN MIDWIFERY PRACTICE.}

BY W. H. BORHAM, Esq., M.R.C.S., SENIOR SURGEON TO THE PADDINGTON DISPEXSARY.

THE following obstetrical cases may prove interesting to the profession: In one case, the labour was complicated with an epidemic disease; in the other, with a constitutional disease. The cerebral and spinal functions were differently influenced in each case: in the first we had an exalted cerebral action, with decreased rootor power; in the latter, an exalted motor power with a total loss of the cerebral function. The third case is one of a very unusual presentation.

CASE 1.--Parturienti Rosalia.-Mrs. S-, a fine, dark young woman, aged eighteen years, the wife of a foreigner, was safely delivered of her second child, a female, at the eighth month term, on Oct. 3rd, at one P.M., after a natural labour of six hours' duration. On calling the following day, I found her with a swollen, scarlet face, very feverish, with a sore throat and strawberry tongue, and a pulse of 120. Her milk had not yet made its appearance; the after-pains and lochia were then natural, and she had micturated freely. I prescribed a saline aperient with antimony, a mustard poultice to the throat, and the usual gruel diet.

Oct. 5th. - Face less swollen; had been delirious a little in the night; the throat better, but the rash all over the chest and body; pulse 120. Her bowels have been relieved, and she micturated; discharge natural and apparently healthy. To continue the saline without the aperient. No milk, and infant well.

6th.-Pulse 130; complete prostration; breathing rather laborious, but I could not detect any abnormal pectoral sounds; pain in the pelvis; lochia entirely and suddenly stopped, and urine suppressed; no milk. Ordered a turpentine and bran poultice to the abdomen; calomel and opium every two hours, and chlorate of potash to the mixture. Eleven P. M. : A sudden change for the worse. I gave her ammonia and chloric ether. She was sensible, and in no pain, but was evidently sinking; and she died on the 7 th, four days after her confinement, the case assuming, the last twelve hours, all the virulence of puerperal malignant fever.

Remarks. - Cases of this kind, complicated with any epidemic disease, become of the most dangerous character. Whether it be small-pox, measles, scarlet fever, or any other sporadic complaint, where the blood becomes influenced with a morbific poison, I have invariably found the milk first suppressed; then follows purulent metritis; then peritonitis, with fever of a low kind, with an exalted imagination and bright glassy eye, attended with little or no pain, which speedily terminates in death.

This case is interesting, inasmuch as we are led to ask whether the premature birth was caused by the mother's blood being impregnated with the scarlatinal poison? Such I think was the case, as she was a strong, healthy-looking woman, and would, in all probability, have gone her full term, had she been proof against the contagion.

There had been scarlet fever in the house for some time, and her little boy was then suffering with it, and she was under its incubation some time before her confinement, as her mother told me she complained of feeling ill, and had a slight sorethroat some days prior. The infant, since its birth, has had no symptoms of the fever; and this leads me to ask if the child could not have had intra-uterine rosalia? We know small-pox and syphilis are conveyed through the medium of the maternal blood to the foetus in utero, and therefore why should we not have scarlet fever so engendered ?

Dr. Meigs, in the work he has lately published in America, has discussed the subject of child-bed fever, in which he denies that it is caused primarily by a blood disease, but gives to it a local origin. His own actual experience in such cases has not been very extensive, and hardly sufficient to warrant his inculcating such a doctrine to his pupils.

There is no doubt that many cases are ushered in through a local origin by a communicable cause, and on the other hand, and I believe far the majority, receive the poison into the blood from a vitiated atmosphere, of an epidemic or foul character Would Dr. Meigs affirm that the above case was one of a local origin, with constitutional symptoms following? Was it not a case of primary blood disease? He does not record in his book a case complicated with scarlet fever in child-bed, and, had he seen one, I doubt not but that his views would be open to a reconciliation with child-bed fever, and its causation by primary blood disease.

During my attendance upon this case, I attended the same week several midwifery cases, and visited daily some thirteen or fourteen cases of scarlet fever, but to no lying-in woman did I communicate any disease, either by infection or contagion.

The above case demonstrates the lessened and depressed state of the spinal functions and the activity of the cerebral; the following will illustrate the reverse, depressed cerebral power and spinal activity.

CASE 2. - Parturient Convulsions. - Mrs. W- aged thirty. seven, a delicate little woman, has been an invalid and subject to fits from girlhood. On April 29th, at four P.M., she was suddenly attacked with convulsions, She was then at the seventh month of pregnancy with her first child. The fit was of the usual character-stertorous breathing, foaming at the mouth, stiffness of the extremities, beating of the temporal arteries, and congestion of the cervical veins, teeth clenched, twitching of the facial muscles, mouth drawn on one side, \&c.: then followed a state of repose alternately with the fits. One pint of blood was extracted from the arm, cold applied to the head, with calomel and colocynth given immediately. The next morning she remained in the same state, the os uteri in its normal position and undilated. An enema unloaded the rectum, and no relief being obtained I sought the assistance and advice of Jr. Tyler Smith, as to the eligibility of indncing premature labour. Dr. Smith concurred that it was desirable labour should be brought about by artificial means, the patient having been twenty hours in a state of alternate convulsions and coma, without any prospect of relief. The membranes were forthwith punctured at twelve meridian, with a stilette, by Dr. Tyler Smith, and five grains of calomel, and one of tartar emetic, given instantly, and a grain of tartar emetic alone placed upon the tongne every half-honr. Pulse 115 ; reflex motor uterine action soon set in, and upon examination at five P. M., the os uteri had dilated to about the size of a fourpenny-piece, the vertex presenting, her fits still continuing very severe. At eight P.M. the convulsions increased in intensity, and $I$ abstracted a pint more blood, which quieted them, and she swallowed, for the first time, a little gruel. The labour-pains became now more frequent and powerful; the vertex was lodging on the symphysis of the pubes, and the os being fully dilated, I dislodged it with the vectis, and forced the os frontis into the concavity of the sacrum, and delivery was then speedily accom. plished, on May 1st, at four A.M., sixteen hours after puncturing the membranes. The child's animation was suspended, but speedily recovered on the application of the usual remedies, and the placenta soon followed. The fits still continued for 
some hours after the child was born, and I again bled her; also applied leeches to the temples; had her hair cut short, and mustard cataplasms to the feet and breasts. This treatment completely subdued them, and the next day she was partially conscious, pulse 100 ; lochia natural ; bowels costive ; abdomen tender. To take a dose of castor oil directly, calomel every two hours, and a mixture with sulphuric ether and henbane. The child suffers from fits.

3rd. - Has had no sleep for forty-eight hours, and has an insane look about her; to take half a grain of morphia every two hours until sleep is produced.

4th. -Slept well, more rational, and better in every respect; and she continued to get better every day, but displayed a silliness and childishness in her manner and actions, which continue to this day. The child died in a fit when a few days old.

Remarlis. - No cases are so embarrassing to the acconcheur as those connected with the epileptic or apopleptic convulsions. I have no doubt many cases of the hysterical type have been mistaken for the above, for the slender and inadequate remedies which have effected their cures wonld certainly lead us to believe they were not of the true cast, as nothing but very active treatment will save them.

From obstetrical records we learn that more than half die who have been attacked thus during labour, but this estimate will not bear a comparison with the experience of more recent times; it is not nearly so large, modern practice baving stamped the treatment of this disease with much improvement.

The preceding case is one of singular interest, inasmuch as the patient was fifty-eight hours in alternate paroxysm of conrulsions and coma, without a single lucid interval, and during that time she was sixteen hours in labour, which was commenced and terminated without her knowing she had had a single labour pain or had given birth to a child The fits did not cease upon the termination of labour, but continued hourly for seven hours after the birth of the child, until the active treatment of the 2nd of May was resorted to, which entirely and at once quelled them.

The history of her family gave a tendency to insanity, and the appearance she displayed rendered her case anxious; but the morphia checked her restlessness and indnced a marked benefit.

The treatment of inducing premature labour was the best that could have been adopted ; it caused a revolutionary change in her whole system, and possibly drained the brain of much superfluous blood. The blood drawn did not show signs of inflammation, nor did the urine show any traces of albumen.

Was this a case of convulsions primarily excived by pregnancy? The patient was a sensitive rroman, had had previous fits, had much domestic reverse, a sick husband, insufficient food, and over-work; these, I think, were the exciting causes, and not utero-gestation. Nevertheless, her cure was to be effected only by emptying the uterus, and thus relieving the brain. I have attended this patient in a subsequent confinement, when she had a natural labour, unattended by any symptoms of a convulsive character.

Case 3.-An Unusual Presentation.-Mrs. B-, aged thirty-two, had had three children, youngest two years old, was taken in labour on September 4 th, at 7 P.M. At 2 A.M. I received a message to attend, and finding the presentation was beyond ascertaining by digital examination, I requested that I might be sent for when the pains became more severe, which was six hours after, when I found the foot, hand, and head presenting as follows:--The right foot was fixed posteriorly, in the hollow of the sacrum, the vertex Jying most anteriorly on the symphysis of the pubes, and the right hand between the two. I endearoured to make a footling case by turning; and although I readily introduced my hand into the uterus, I could not do so, owing to the body of the uterus contracting powerfully on the child, in the manner of the hour-glass contraction.

I asked my friend and neighbour, Mr. J. C. Langmore, to see the case with me, and it was thought desirable to draw the foot external, and secure it with a piece of tape. This we did, but, owing to the peculiar action of the uterus during a pain, instead of expelling, it retractod the foot. We gave her thirty drops of Battley's solution, then pushed the hand and head up, making traction upon the foot, and by this means it soon resolved itself into a footling case, but from the enormous size of the child, it was an hour before it was expelled, of course dead. The funis was twisted round the child's neck, and the placenta soon followed, with considerable hæmorrhage. She did well.
Remarks. - The child's position in the uterus, from being so doubled up, possibly caused the hour-glass contraction, and prevented the usual expelling power. The Battley's solution allayed this irregular state, and gave us an opportunity of making an easy and safe evolution of the child. Chloroform would have been resorted to if Battley's solution had failed.

Cambridge-terrace, Hyde-park, 1856.

REPORT OF A

CASE OF LABOUR COMPLICATED BY FRACTURED PELVIS AND DISLOCATED HIP OF OLD STANDING.

\section{By T. APPLEBY STEPHENSON, EsQ., M.R.C.S. ENG.}

WHILE assisting a medical friend in Leicestershire, (W. G. Palmer, Esq., of Loughborough, I was called at noon on the 16th of October, to Mrs. C--_, a stout, florid-looking woman, whom $I$ found in labour of her fifth child. The pains had been intermittent and grumbling for two days; had changed their type early that morning, but not assumed the distinct character of true labour ones till eight o'clock A.M. A midwife being present, the medical attendant was not sent for till it appeared requisite. The patient seemed nervous and much excited, her countenance and tone evincing acute anxiety ; for this I thought there must be a cause, but all of her previous history I then learnt was, that six years had elapsed since her last confinement, soon after which she met with an accident; and some one had subsequently said she must never have another child or it would "be the death" of her, though she had always, as in this case, gone her natural time, had easy and good labours, and invariably made rapid recoveries.

On the occurrence of a strong pain, I made an examination, detecting at the time an obstacle, and perceiving an unnatural shrinking on the part of the patient; the os uteri was fully dilated, but as yet the membranes were unruptured, and through them a natural head presentation was plainly felt. The pains were strong and at regnlar intervals, "the bag of the waters" bursting about two P. M., yet still the head made very little progress in its descent. In a second and more careful examination, the passages were found narrowed behind and below the pubes, by an increase of hard or bony substance, diagnosed and afterwards confirmed as the remains of an old fracture, apparently between the right os pubis and the symphysis; there had also been an ill- or non-reduced dislocation of the right hip upwards aid forwards on the pubes, where an imperfect false joint was made, the leg still remaining three-quarters of an inch shorter than the left, and the foot pointing outwards. The pains continued forcible and frequent enough, yet made little advancement; but the presentation changed from the third to that of " the forehead to the pubes." Up to this time (five o'clock P.M.) I hoped that my patient might still be delivered naturally, but foreseeing in her excitement, \&c., symptoms of exhaustion, $I$ explained to her friends the peculiarity of the case, and that owing to the mechanical obstruction instruments would at least be requisite. To satisfy them and myself, I sent for Mr. Palmer, after whose minute and careful examination we determined first to try the forceps, though we had fears that craniotomy would have to be resorted to ; the locking was, with a little difficulty, accomplished, and by more than ordinary care, time, and gentle traction, Mrs. C. was at ten minutes past six P.M. safely delivered of a male child. The head was smaller in its diameters than usual, or the infant's life conld not have been thus saved; in this case it was only by getting up artificial breathing and the other restorative means. The face was of course much reddened and contused. Fortunately the perinæum of the mother was not in the least rigid, and there was plenty of room in the sacral cavity; the placenta was brought away from the posterior part of the vagina. Fifteen minutes afterwards the uterus contracted steadily but slowly, and the patient only lost about twice as much blood as ordinarily; an hour after. wards I left her enjoining especial precautions. Ordered a draught with fifty-five drops of tincture of opium, and in the morning found she had slept a little.

The draught was repented next night, and after the simple treatment, the bowels moved on the third day. No peritonitis or other malady supervened, and she made (for her case) a quick recovery, being able to command constant attention.

Of her previous history, I learnt the following :- Six years aro, while living in Lincolnshire, she was crossing a road, when 